\title{
Protein losing enteropathy-hepatic fibrosis syndrome in Saguenay-Lac St-Jean, Quebec is a congenital disorder of glycosylation type lb
}

\author{
S Vuillaumier-Barrot, C Le Bizec, P de Lonlay, A Barnier, G Mitchell, V Pelletier, \\ C Prevost, J M Saudubray, G Durand, N Seta
}

J Med Genet 2002;39:849-851

C ongenital disorders of glycosylation (CDG) are newly described inborn errors of metabolism involving glycan moiety synthesis. CDG Ib is caused by a deficiency of cellular phosphomannose isomerase (EC 5.4.2.28) (1-4 $^{1-}$ resulting from the presence of mutations in the corresponding gene, $M P I^{56}$ on chromosome 15 (mutation database: http:// www.med.kuleuven.ac.be/cdg/). CDG Ib differs clinically from the other CDGs. It has no neurological symptoms, presenting mainly with hepatic and gastrointestinal symptoms ${ }^{2378}$ and is thought to be underdiagnosed. Fewer than 20 CDG Ib patients have been reported. Importantly, CDG Ib is treatable by oral mannose supplementation, in contrast with other CDGs. ${ }^{39}$

The syndrome of intractable diarrhoea of infancy was first defined in 1968 by Avery et al. ${ }^{10}$ In 1986, a syndrome of intractable diarrhoea associated with severe protein losing enteropathy, hypoglycaemia, and congenital hepatic fibrosis, was described in four infants from the Saguenay-Lac St-Jean (SLSJ) region of north eastern Quebec, Canada. All died before 21 months of age. ${ }^{11}$

In retrospect, their symptoms resembled those of CDG Ib. In this article we describe the testing of parents of these children and report on a new French family with CDG Ib. The FrenchCanadian parents were all heterozygous for the same deleterious MPI mutation as found in the new French patient, proving that the SLSJ patients in fact suffered from CDG lb.

\section{PATIENTS AND METHODS \\ Patients}

The clinical presentation of the SLSJ infants was previously described by Pelletier et al. ${ }^{11}$

The new French patient with CDG Ib, whose family originates from Nantes (Brittany, France) and whose parents have no known consanguinity, presented with diarrhoea related to protein losing enteropathy with oedema, hypoalbuminaemia, hyponatraemia, and recurrent hypoglycaemia. No liver cytolysis was noted. Hypocholesterolaemia was found and clotting tests showed thrombocytosis. Coagulation factor XI was decreased, resulting in an increased prothrombin time. The patient had a typical CDG I profile on western blotting of serum glycoproteins. ${ }^{12}$ Blood leucocyte PMI activity was deficient $(0.5 \mathrm{U} / \mathrm{g}$ total protein).

\section{Methods}

DNA samples were not available from the affected SLSJ children, of whom all known cases have died; no new cases have been identified since the initial publication. ${ }^{11}$ EDTA blood samples were obtained from three parents of SLSJ patients, both parents of one affected child, and the mother of a second patient; the father of the second patient, who was not sampled, was distantly related to the mother (consanguinity $1 / 64$ ). All originated from the SLSJ region. Control blood samples were simultaneously obtained from two unrelated

\section{Key points}

- Congenital disorder of glycosylation with hereditary phosphomannose isomerase (PMI) deficiency (CDG lb) is a very rare disease, probably underdiagnosed. A fatal syndrome of protein losing enteropathy and congenital hepatic fibrosis described in 1986 in the Saguenay-Lac St-Jean (SLS) region of Quebec, Canada, clinically resembles CDG lb, with intractable diarrhoea, hypoglycaemia, hepatomegaly, vomiting, and malnutrition.

- This prompted us to study PMI activity and the PMI encoding gene, MPI, in blood samples from three parents of two of the affected Canadian children. All the parents had partial deficiency of leucocyte PMI activity. MPI gene sequencing showed that all three were heterozygous for the same mutation in exon $7, \mathrm{R} 295 \mathrm{H}$ (G884A).

- Meanwhile, a newly diagnosed French CDG lb patient was found to be homozygous for R295H and also for nearby polymorphic markers. Interestingly, an identical variant was also found for each marker on at least one chromosome of each of the SLSJ parents.

- This indicates that the SLSJ syndrome is a variant of CDG lb. Since CDG lb is treatable by oral mannose supplementation, awareness of this condition is imperative to allow early diagnosis and treatment.

healthy adults, to control for possible loss of activity during transport. Samples were also obtained from the French CDG Ib patient and his parents.

Cytosolic PMI activity was assayed in blood mononuclear cells, according to van Schaftingen and Jaeken. ${ }^{13}$

DNA was isolated from fresh blood. The eight MPI exons were sequenced from genomic DNA after PCR with intronic primers designed from published DNA sequences ${ }^{6}$ (GenBank AF227216, AF227217, AF227218). Primer sequences are available on request. Sequencing was performed on an ABI PRISM (Perkin Elmer). The R295H (G884A) mutation creates a NlaIII restriction site, which conveniently allowed confirmation of $\mathrm{R} 295 \mathrm{H}$ by restriction digestion of the exon 7 amplicon and separation by electrophoresis in a 10\% bis-acrylamide gel (fig 1).

Haplotype analysis was performed on the Canadian parents and the French CDG Ib family. All were typed for the extragenic microsatellite markers D15S984, D15S1026, D15S114, and D15S818. Primer sequences were obtained from the Genome DataBase. The PCR products were analysed on an ABI PRISM (Perkin Elmer). Three intragenic single nucleotide polymorphisms, IVS3 + 15A/G, IVS5 +9G/A, and Al131G (V377V), were screened by sequencing of exons 3,5 , and 8 , respectively. 


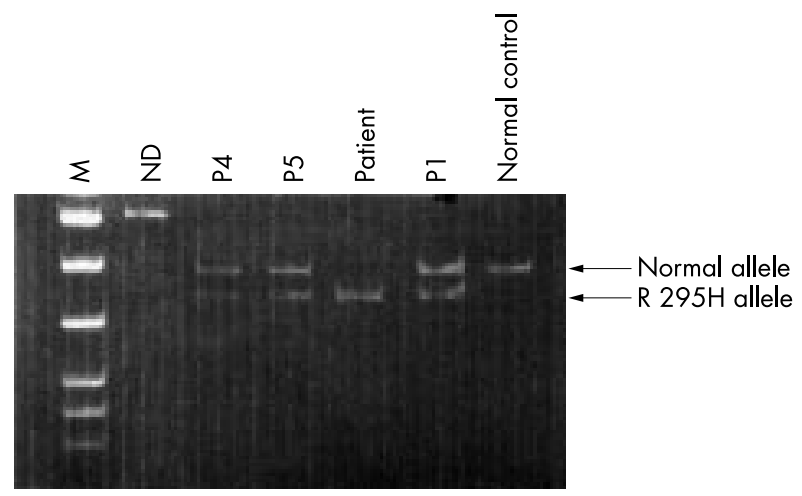

Figure 1 RFLP analysis of exon 7. The R295H mutation creates a Nlalll restriction site. CDG lb parents (P4 and P5) and a SLSJ parent (P1) are heterozygous R295H/N. The French CDG lb patient is a R295H homozygote. The amplicon contains a constant Nlalll site that serves as a control for completeness of digestion.

$N D=$ non-digested fragment.

\section{RESULTS AND DISCUSSION}

We report on a new French patient with CDG Ib and show that the SLSJ patients suffered from CDG Ib. To date, all reported CDG Ib patients have had similar clinical presentations with protein losing enteropathy, recurrent hypoglycaemia, and liver symptoms. ${ }^{7}$ We confirm in this report the clinical homogeneity of CDG Ib.

Blood leucocyte PMI activity of the three Canadian parents was reduced $(2.7,2.0$, and $1.6 \mathrm{U} / \mathrm{g}$ total protein), compared to the Canadian (5.5 and $6.0 \mathrm{U} / \mathrm{g}$ total protein) and local (8.2 \pm $3.3 \mathrm{U} / \mathrm{g}$ total protein) adult controls.

Sequencing of the PMI encoding MPI cDNA of the Canadian parents showed that all were heterozygous for a single mutation in exon 7, R295H (G884A). This mutation had not been previously reported. During this period, we found that the French patient was homozygous for $\mathrm{R} 295 \mathrm{H}$ and that his parents were heterozygous. No other sequence changes were detected in the coding regions of the samples tested, aside from a previously reported synonymous polymorphism at residue 377 (see below). The $\mathrm{R} 295 \mathrm{H}$ mutation screened by restriction analysis was not found in 50 French controls and the two Canadian controls, indicating that it is not a polymorphism.

Haplotype analysis of the MPI locus indicated that the Canadian parents are identical for at least one allele for the extragenic microsatellite markers D15S984, D15S1026, D15S114, and D15S818 and three intragenic single nucleotide polymorphisms IVS3 $+15 \mathrm{~A} / \mathrm{G}$, IVS5 $+9 \mathrm{G} / \mathrm{A}$, and All31G (table 1). However, all three Canadian parents originate from the north eastern part of Quebec ${ }^{11}$ and our data suggest that the parents share the same ancestral chromosome showing a founder effect in the SLSJ region. Of note, the French CDG Ib patient, a R295H homozygote, was also homozygous for the same variant at each of the markers tested that was shared by all the SLSJ parents. It is tempting to speculate that the SLSJ patients may have inherited the same ancestral CDG Ib chromosome as the French patient. To pursue this hypothesis, it would be necessary to perform extensive segregation analysis to determine whether variants exist in cis on the SLSJ CDG Ib chromosomes (table 1). These studies would be of interest because identity by descent has rarely been documented for mutations found in France and the SLSJ population, even though the SLSJ population is of French descent.

The arginine residue at position 295 is conserved in all species studied to date (S typhimurium, E coli, Candida albicans, Saccharomyces cervisiae, A nidulans, and human MPI) consistent with the notion of a functional effect of the mutation. ${ }^{5}$

Together, these data indicate that at least two of the SLSJ patients reported by Pelletier et $a l^{11}$ suffered from CDG Ib. Given the clinical similarity of the patients, and their common geographical origin from the SLSJ region that is known to have a strong founder effect, shown, for instance, for hepatorenal tyrosinaemia ${ }^{14}$ and cystic fibrosis, ${ }^{15}$ it is likely that the great majority of CDG Ib patients from the SLSJ will be R295H homozygotes. Identification of the $\mathrm{R} 295 \mathrm{H}$ mutation as the cause of the enteropathy-hepatic fibrosis syndrome in SLSJ children will allow for other studies to assess the frequency of this mutation in the SLSJ and surrounding regions. The lack of new cases after the report of the first cluster over 15 years ago raises questions about the frequency and distribution of the mutant allele and about disease expressivity. These questions can now be explored directly. They are particularly important because CDG $\mathrm{lb}$ is treatable but can be fatal if untreated, and awareness of this disease is imperative to allow early diagnosis and therapy. CDG Ib should be considered in the differential diagnosis of patients with unexplained hypoglycaemia, chronic diarrhoea, liver disease, or coagulopathy, particularly in patients of SLSJ origin.

\section{ACKNOWLEDGEMENTS}

This work was supported by the Réseau de recherche sur les CDG (INSERM/AFM) 4MR29F.

\section{Authors' affiliation}

S Vuillaumier-Barrot, C Le Bizec, A Barnier, G Durand, N Seta, Biochimie A, Hôpital Bichat-Claude Bernard, AP-HP, 75877 Paris Cedex 18, France

P De Lonlay, J M Saudubray, Département de Pédiatrie, Hôpital Necker, AP-HP, 75743 Paris Cedex 15, France

G Mitchell, V Pelletier, Service de Génétique Médicale, Hôpital

Sainte-Justine, 3175 Côte Sainte-Catherine, Montréal, Canada

C Prevost, Service de Génétique, Centre Hopitalier de la Sagamie, Canada

Correspondence to: Dr N Seta, Laboratoire de Biochimie A, Hopital Bichat-Claude Bernard46 Rue Henri Huchard, 75877 Paris Cedex 18, France; nathalie.seta@bch.ap-hop-paris.fr

Table 1 Haplotypes of SLSJ parents (P1, P2, and P3) and the French CDG lb parents (P4 and P5) and patient. Alleles in bold represent the alleles consistent with linkage desequilibrium with $\mathrm{R} 295 \mathrm{H}$. The five parents share at least one polymorphic variant at each tested site, the same as that found in the homozygous state in the French CDG Ib patient

\begin{tabular}{lllllllll}
\hline & Mutation & D15S818 & D15S1026 & IVS3 +15 A $\rightarrow$ G & IVS5 +9 G $\rightarrow$ A & A1131G (V377) & D15S114 & D15S984 \\
\hline P1 & R295H/N & $148-156$ & $199-199$ & GA & GA & GA & $180-182$ & $129-147$ \\
P2 & R295H/N & $148-166$ & $199-209$ & GA & GA & GA & $180-182$ & $129-139$ \\
P3 & R295H/N & $148-148$ & $199-209$ & GA & GA & GA & $180-182$ & $129-139$ \\
& & & & & GG & GG & $178-182$ & $129-141$ \\
P4 & R295H/N & $148-148$ & $199-199$ & AA & GG & GG & $182-182$ & $129-135$ \\
P5 & R295H/N & $148-148$ & $199-211$ & AA & GA & GG & $182-182$ & $129-129$ \\
Patient & R295H/R295H & $148-148$ & $199-199$ & AA & GG & & &
\end{tabular}




\section{REFERENCES}

1 de Koning TJ, Toet M, Dorland L, de Vries LS, van den Berg IE, Duran $M$, Poll-The BT. Recurrent nonimmune hydrops fetalis associated with carbohydrate-deficient glycoprotein syndrome. J Inherit Metab Dis 1998;21(6):681-2

2 Niehues R, Hasilik M, Alton G, Korner C, Schiebe-Sukumar M, Koch HG, Zimmer KP, Wu R, Harms E, Reiter K, von Figura K, Freeze HH, Harms HK, Marquardt T. Carbohydrate-deficient glycoprotein syndrome type $\mathrm{lb}$. Phosphomannose isomerase deficiency and mannose therapy. $J$ Clin Invest 1998;101:1414-20.

3 de Lonlay P, Cuer $M$, Vuillaumier-Barrot $S$, Beaune $G$, Castelnau $P$, Kretz M, Durand G, Saudubray JM, Seta N. Hyperinsulinemic hypoglycemia as a presenting sign in phosphomannose isomerase deficiency: a new manifestation of carbohydrate-deficient glycoprotein syndrome treatable with mannose. J Pediatr 1999;135:379-83.

4 Jaeken J, Matthijs G, Saudubray JM, Dionisi-Vici C, Bertini E, de Lonlay $P$, Henri H, Carchon H, Schollen E, Van Schaftingen E. Phosphomannose isomerase deficiency: a carbohydrate-deficient glycoprotein syndrome with hepatic-intestinal presentation. Am J Hum Genet 1998;62:1535-9.

5 Proudfoot AE, Turcatti G, Wells TN, Payton MA, Smith DJ. Purification, cDNA cloning and heterologous expression of human phosphomannose isomerase. Eur J Biochem 1994;219:415-23.

6 Schollen E, Dorland L, de Koning TJ, Van Diggelen OP, Huijmans JG, Marquardt T, Babovic-Vuksanovic D, Patterson M, Imtiaz F, Winchester B, Adamowicz M, Pronicka E, Freeze H, Matthijs G. Genomic organization of the human phosphomannose isomerase (MPI) gene and mutation analysis in patients with congenital disorders of glycosylation type lb (CDG-lb). Hum Mutat 2000;16:247-52.

7 Babovic-Vuksanovic D, Patterson MC, Schwenk WF, O'Brien JF, Vockley J, Freeze HH, Mehta DP, Michels VV. Severe hypoglycemia as a presenting symptom of carbohydrate-deficient glycoprotein syndrome. $J$ Pediatr 1999;135:775-81.
8 de Koning TJ, Dorland L, van Diggelen OP, Boonman AM, de Jong GJ, van Noort WL, De Schryver J, Duran M, van den Berg IE, Gerwig GJ, Berger R, Poll-The BT. A novel disorder of $\mathrm{N}$-glycosylation due to phosphomannose isomerase deficiency. Biochem Biophys Res Commun 1998;245:38-42.

9 Westphal V, Kjaergaard S, Davis JA, Peterson SM, Skovby F, Freeze $\mathrm{HH}$. Genetic and metabolic analysis of the first adult with congenital disorder of glycosylation type lb: long-term outcome and effects of mannose supplementation. Mol Genet Metab 2001;73:77-85

10 Avery GB, Villavicencio O, Lilly JR, Randolph JG. Intractable diarrhea in early infancy. Pediatrics 1968;41:712-22.

11 Pelletier VA, Galeano N, Brochu P, Morin CL, Weber AM, Roy CC. Secretory diarrhea with protein-losing enteropathy, enterocolitis cystica superficialis, intestinal lymphangiectasia, and congenital hepatic fibrosis: a new syndrome. J Pediatr 1986;108:61-5.

12 Seta N, Barnier A, Hochedez F, Besnard MA, Durand G. Diagnostic value of Western blotting in carbohydrate-deficient glycoprotein syndrome. Clin Chim Acta 1996;254:131-40.

13 Van Schaftingen E, Jaeken J. Phosphomannomutase deficiency is a cause of carbohydrate-deficient glycoprotein syndrome type I. FEBS Lett 1995; 377:318-20.

14 Grompe M, St-Louis M, Demers SI, al-Dhalimy M, Leclerc B, Tanguay RM. A single mutation of the fumarylacetoacetate hydrolase gene in French Canadians with hereditary tyrosinemia type I. N Engl J Med 1994;331:353-7.

15 De Braekeleer R, Daigneault J, Ferreira-Rajabi L, Gerde M, Lamoureux L, Aubin G, Simard F, Fujiwara TM, Morgan K. Cystic fibrosis mutations in French Canadians: three CFT mutations are relatively frequent in a Quebec population with an elevated incidence of cystic fibrosis. Am J Med Genet 1992;42:360-4

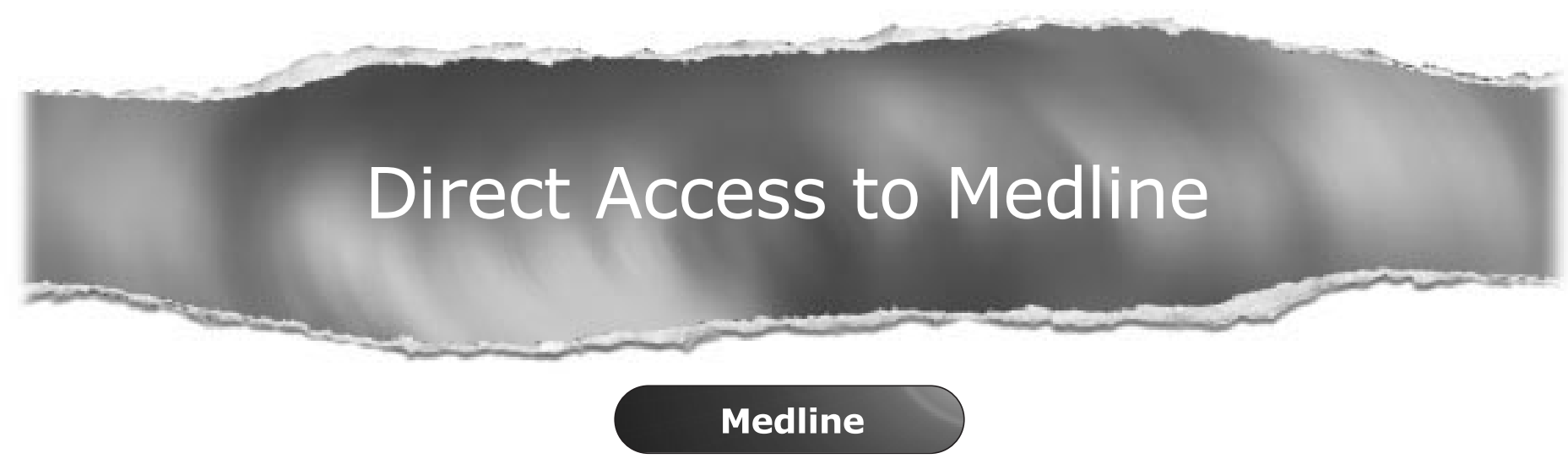

Link to Medline from the homepage and get straight into the National Library of Medicine's premier bibliographic database. Medline allows you to search across 9 million records of bibliographic citations and author abstracts from approximately 3,900 current biomedical journals.

\section{www.jmedgenet.com}

